\title{
La adjetivación de la justicia desde la perspectiva de la teoría de la acción
}

Duvan Fernando Valencia García

Universidad Pontificia Bolivariana, Colombia

Secretaría de Educación de Antioquia, Colombia 


\title{
La adjetivación de la justicia desde la perspectiva de la teoría de la acción*
}

\begin{abstract}
Resumen: el presente artículo pretende ser una reflexión que desde la teoría de la acción contribuya al estudio del problema de la justicia; esta puede verse sumergida en una especie de encrucijada conceptual al ser objeto de estudio tanto de la filosofía moral como de la filosofía política. El artículo reflexiona sobre dos cuestiones fundamentales: la primera gira en torno al lugar de reflexión que ocupa la justicia en la filosofía y la imposibilidad de definirla, porque se considera como una virtud simple, y cualquier intento de definición se enmarca en lo que George Moore llamó falacia naturalista. La segunda intenta poner en conversación la teoría de la acción, especialmente la propuesta de Hannah Arendt, con la teoría de justicia de John Rawls, de modo que sea posible determinar cómo la acción de los individuos permite adjetivar la justicia en lo justo, pero no definirla.
\end{abstract}

Palabras clave: justicia, teoría de la justicia, teoría de la acción, adjetivación de la justicia, sociedad política.

\section{The adjustment of justice from the perspective of the theory of action}

\begin{abstract}
: this article aims to be a reflection that, from the theory of action, contributes to the study of the problem of justice, which can be immersed in a kind of conceptual crossroads as it is the object of study of both moral philosophy and political philosophy. The article reflects on two fundamental questions: the first revolves around the place of reflection that justice occupies in philosophy and the impossibility of defining it, because it is considered as a simple virtue, and any attempt at definition is framed in what George Moore called naturalistic fallacy. The second will attempt to discuss the theory of action, especially Hannah Arendt's proposal, with the theory of justice of John Rawls, so that it is possible to determine how the action of individuals allows the justice to be justified, but not to define it.
\end{abstract}

Keywords: justice, theory of justice, theory of action, adjectives of justice, political society.

Fecha de recepción: 21 de noviembre de 2018

Fecha de aceptación: 17 de julio de 2019

Forma de citar (APA): Valencia-García, D. (2020). La adjetivación de la justicia desde la perspectiva de la teoría de la acción. Revista Filosofía UIS, 19(1), DOI: 10.18273/revfil.v19n1-2020006

Forma de citar (Harvard): Valencia-García, D. (2020). La adjetivación de la justicia desde la perspectiva de la teoría de la acción. Revista Filosofía UIS, 19(1), 185-202.

Duvan Fernando Valencia García: colombiano. Magíster en Estudios Políticos. Doctorando en Filosofía en la Universidad Pontificia Bolivariana.

ORCID iD: orcid.org/0000-0003-1229-976X

Correo electrónico: dufevaga@gmail.com

*Artículo de reflexión derivado de investigación. 


\section{La adjetivación de la justicia desde la perspectiva de la teoría de la acción'}

\section{Introducción}

Constantemente el hombre experimenta situaciones que lo hacen pensar en la justicia. Nos enfrentamos a circunstancias en nuestra vida diaria donde se pone a prueba la carga moral que dirige nuestro actuar; elegimos, hablamos, actuamos o callamos, guiados por nuestros valores y, en algunos casos, conscientes de que nuestras decisiones tendrán un efecto en el otro.

En la mayoría de los casos en que hacemos el bien, admitimos que actuamos justamente, sin embargo, dicha admisión no implica que hayamos aprehendido algunos de estos valores, es decir, difícilmente nuestros actos servirán para definir la bondad o la justicia, cuando mucho, podremos considerar ese acto en particular como bueno o justo, determinado por nuestra concepción del bien y nuestro sentido de la justicia.

Este texto proporciona elementos al lector, que permiten un acercamiento reflexivo al problema que enmarca la justicia como una virtud materializable frente a los otros. Es claro que nadie es justo o injusto consigo mismo, ya que la materialización de lo justo o injusto requiere por lo menos de otra persona, por lo tanto, para hablar de una acción justa esta deberá producir una afectación en el otro y, en consecuencia, a esa afectación se le dará un juicio de valor como justa o injusta, sin poder hacer otro tipo de análisis.

\section{La justicia entre la filosofía moral y la filosofía política}

Desde la Grecia clásica, el estudio de la justicia ha sido una preocupación para la filosofía, aunque las primeras reflexiones se encuentran en los diálogos platónicos especialmente en La República, es Aristóteles el gran precursor de la reflexión filosófica sobre la justicia, a la cual le dedicará en Ética a Nicómaco un

${ }^{1}$ Este artículo es un avance parcial de la tesis doctoral en filosofía, titulada De lo justo a la justicia. Debate entre la filosofía moral y la filosofía política, que actualmente desarrolla el autor en la Universidad Pontificia Bolivariana de Medellín, Colombia. 
capítulo entero. Este es, sin duda, el primer intento filosófico por definir qué es aquello a lo que llamamos justicia. En ambos filósofos clásicos, la justicia está determinada por el actuar del hombre en relación con el valor de la bondad. Se entenderá, en un primer momento, que la justicia es el resultado del actuar del hombre bueno.

La justicia es un concepto polisémico, existen por lo menos tres acepciones de ella: "dar a cada persona lo que se merece", "dar a cada quién lo que se debe" y "hacer lo correcto". A partir de estas acepciones surgen las siguientes preguntas: ¿qué es lo que se merece?, ¿qué es lo que se debe?, ¿qué es lo correcto?, incluso cabe una pregunta más general: ¿a quién le corresponde dar a cada quién lo que se merece, dar lo que se debe, o hacer lo correcto?, la respuesta a estos interrogantes se buscó desde Platón hasta Kant en la filosofía moral, lo que condujo a reducir la concepción de la justicia al juicio moral que se les hace a nuestras acciones.

El problema de la filosofía moral consiste, precisamente, en reducir la concepción de la justicia al juicio que reciben nuestras acciones como justas o injustas, dependiendo, incluso, de las circunstancias que motiven el obrar de una manera y no de otra. Los dilemas morales son un claro ejemplo de esto. Al no poder determinar con certeza cuál de las dos acciones resultará justa, debido a que están sometidas al campo individual, necesariamente una será justa y la otra resultará injusta, dejando su realización a criterios morales.

Tal es el caso del dilema del tranvía propuesto por Philippa Fott, en el cual usted es el conductor de un tranvía que va sin frenos a alta velocidad, ante usted se encuentran cinco hombres realizando el mantenimiento de la vía, y si no hace nada el tranvía los matará. De pronto se da cuenta de que hay otra vía a la derecha, donde se encuentra un solo hombre trabajando, puede halar una palanca y desviar el tranvía hacia donde está el hombre solo. La decisión de halar o no la palanca indiscutiblemente producirá muerte, ¿qué haría?; para la mayoría puede ser preferible halarla, ya que morirá una sola persona, lo cual considerarían el menor mal posible, pero si se introduce la variante de ser esa persona un ser amado, seguramente la decisión de no halar la palanca y matar a las cinco personas sería la conveniente. Para Michael Sandel, la diferencia no estará en las consecuencias sino en la intención de halar o no la palanca "la diferencia moral, quizá, no estriba en las consecuencias para las víctimas - ambas mueren-, sino en la intención del que decide" (Sandel, 2011, p. 33).

Este dilema se ajusta a los criterios de la ética normativa y podría ser resuelto desde los argumentos del utilitarismo y de la ética deontológica. Para el utilitarista resultaría conveniente, en el primer caso, halar la palanca, puesto que se maximizaría el bienestar de la mayoría, en el sentido de que se salvarían cinco vidas en vez de una. Sin embargo, en el segundo caso, con la variante de ser la persona que está en la otra vía un ser amado, el utilitarismo resulta inútil, puesto 
que el criterio de máximo bienestar se vería truncado por el sentimiento de amor hacia el ser que se encuentra en la vía y no se halaría la palanca, prefiriendo matar cinco personas, ya que consideraría más justo salvar la vida del ser que ama, aunque para Rawls "una persona justa no está dispuesta a hacer determinadas cosas y, si cede demasiado fácilmente a la tentación, es porque en realidad ya estaba dispuesta" (Rawls, 1995a, p. 513), lo cierto es que, para el caso planteado, incluso un deontológico estaría dispuesto a abandonar sus convicciones morales para salvar la vida de su ser amado; siguiendo a Kant, la máxima que actuaría sería el amor a esa persona, en ese sentido, la ponderación llevaría a comprender que, como imperativo categórico, primero está el amor que la moral.

Lo anterior refleja que la reflexión no se centra en la justicia, sino en lo justa o injusta que podría resultar la acción de halar la palanca. Así entendida, nos vemos de frente con la adjetivación de la justicia. Se muestra, entonces, que lo justo o lo injusto es un juicio de valor referente a un comportamiento o una acción puntual y que, por el contrario, la justicia no puede asimilarse con ese comportamiento ni con esa acción, ya que esta se encuentra en el ámbito de lo general y lo abstracto de la sociedad política, por lo tanto, es necesario hacer una diferenciación entre la justicia y lo justo o lo injusto. Dicha diferenciación se encuentra sustentada en que la justicia se adjetiva en lo justo o lo injusto como juicios de valor que recibe la acción de los individuos. Lo mismo podría ocurrir con otros ejemplos, como el derecho a la legítima defensa, donde asesinar a otra persona se considera justificado si el móvil fue salvar la propia vida.

Vemos que las líneas anteriores sirven para definir y considerar una acción como justa o injusta, correcta o incorrecta, buena o mala, pero no sirven para definir, concretamente, qué es la justicia, ya que no podemos aseverar que siempre ante esas situaciones la mayoría de las personas actuarán de la misma manera y, al momento de juzgar, tendríamos que asegurarnos de no tener dudas sobre las motivaciones que tuvo quien haló o no la palanca.

Sin duda, estamos frente a una situación conducente a establecer los criterios para los juicios morales que nos llevaron a tomar la decisión de halar o no la palanca, en ese sentido "los juicios morales son «razones motivadoras» para actuar; con lo cual se pretende decir que las personas hacen cosas simplemente porque piensan que deben hacerlas" (Foot, 2002, p. 48). Esto es, precisamente, lo que hace evidente el problema de la justicia desde la filosofía moral, pues no existe un carácter universal que permita calificar siempre y en todas las circunstancias las decisiones como buenas o justas, ya que los juicios morales operan como motivadores, "en cuanto a los juicios éticos que afirman que una determinada clase de acción es buena como medio para conseguir un tipo concreto de efecto, ninguno de ellos será cierto con carácter universal" (Moore, 1903, p. 46). Ciertamente, entender la justicia desde la filosofía moral no permite someterla a un proceso de razonamiento lógico, esta no transciende más allá del 
razonamiento moral, por lo tanto "no hay ninguna justicia en sí, ninguna acción buena ni obra justa alguna, que no haga referencia a un modelo de racionalidad práctica" (Maclntyre, 1994, p. 8). Si no es posible realizar un razonamiento lógico de la justicia desde la filosofía moral, debe ser porque la justicia no es una virtud moral sino una virtud política, debido a que solo desde la acción política es posible —al menos en la construcción teórica— definir qué es la justicia.

El origen de la política pone de manifiesto la obligatoriedad inmanente que tiene el hombre de vivir en sociedad, esto lo lleva indiscutiblemente a la interacción que es donde el hombre crea la política, sin embargo, desde del Estado liberal lockeano es posible hablar de la libertad y la igualdad como valores fundantes de una sociedad política, el liberalismo como doctrina tiene como finalidad la igualdad" ${ }^{2}$, en el sentido de que "por naturaleza los hombres son desiguales, por lo tanto necesitan de una institución política para llegar a serlo. Sólo el acto político puede generar igualdad" (Arendt, 1997, p. 22).

Por lo tanto, la política como constructora de la sociedad debe asegurar que esta sea justa, equitativa e igualitaria, en ese sentido "la justicia requiere de políticas que remedien las desventajas sociales y económicas y den a todos equitativamente oportunidades de triunfar" (Sandel, 2011, p. 30). Se debe anotar que concebir la justicia como valor moral remite a la reflexión filosófica de la acción, en esa medida, la justicia es realizable, concretizable, y se presenta como resultado de lo justo o lo injusto; por el contrario, concebir la justicia como un valor político implica una reflexión de la sociedad política y sus instituciones. La justicia es entendida como el telos del Estado, por lo que constituye el ideario que justifica la actuación estatal.

Al trasladar la reflexión de la justicia al campo de la filosofía política, desaparecen esas diferencias de criterios morales, la justicia siguiendo a Hans Kelsen será "una cualidad posible, pero no necesaria, de un orden social que regula las relaciones mutuas entre los hombres" (2014, p. 2). El problema que se plantea en torno a la justicia es darle un lugar propio en la reflexión filosófica, desde la filosofía moral se ha tratado de modo indiferenciado lo justo con la justicia, en la concretización de una acción individual que soporta un juicio ético, precisamente, un hombre podrá ser justo o injusto, pero no podrá hacer justicia, "un hombre es justo si su conducta se adecúa a las normas de un orden social supuestamente justo" (Kelsen, 2014, p. 2).

La postura de Kelsen encierra la solución al problema de la justicia entre la filosofía moral y la filosofía política, así planteado, lo justo pertenece al ámbito individual de la acción moral, por lo tanto, es propio de la filosofía moral, mientras

${ }^{2}$ La igualdad es entendida, en este sentido, como un sinónimo de la justicia. 
que la justicia pertenece al ámbito del orden social, de la sociedad política, por lo que su lugar está en la filosofía política.

\section{La imposibilidad de definir la justicia}

Este apartado se centra sobre la imposibilidad de definir la justicia, debido a que es un concepto simple carente de partes. Si se asume que definir es descomponer en partes, entramos al problema de la indefinición de la justicia, para esto, se relacionará el concepto "falacia naturalista" acuñado por George Moore en el libro Principia Ethica (1903), donde afirma que la finalidad de la ética es lo bueno. Sin embargo, cuando se pregunta iqué es lo bueno?, Moore es enfático en argumentar que lo bueno, por ser una cualidad simple, es indefinible, y cualquier pretensión de definir lo bueno conducirá a la falacia naturalista.

Principia Ethica es un excelente puerto de salida para el pensamiento analítico de la justicia. Generalmente, la justicia está relacionada con hacer el bien, pues no se puede considerar que exista justicia donde hay maldad. Así, cobra sentido la definición hecha por Aristóteles en Ética a Nicómaco de la justicia como "hacer lo correcto", el problema resulta cuando se pretende dar cualidades a la justicia, y se trata de limitarla y definirla, pues esto conduce a un rompiendo de los presupuestos racionales, y da origen a la única pretensión posible: significar la justicia con lo justo. Esta pretensión se relaciona con el concepto de falacia naturalista. La conclusión de Moore es que el concepto del bien es indefinible, puesto que la bondad es una cualidad simple que carece de partes:

\footnotetext{
Si se me pregunta «zqué es bueno?», mi respuesta es que lo bueno es lo bueno. Y eso pone punto final al asunto. O, si se me pregunta «icómo puede definirse lo bueno?», mi respuesta es que no puede definirse (Moore, 1903, p. 29).
}

Ahora, si el objeto de hacer el bien es alcanzar la justicia, resultan aplicables a la justicia, por analogía, las mismas características del bien, ya que se encuentra en el mismo rango axiológico y, como se dijo anteriormente, no se puede concebir la justicia sin la bondad, ni la bondad sin la justicia. Si entendemos la justicia como sinónimo de bien, tendríamos que admitir necesariamente que la justicia, al igual que el bien, también es una virtud simple, por tanto, cuando digo a alguien que haga justicia, realmente le quiero decir: sea justo; pues, al ser la justicia una virtud simple, no es posible saber qué o cuál acción me conduce necesariamente a ella.

Los filósofos analíticos posteriores a Moore "concluían que las proposiciones morales no son cognitivamente significativas, y que su función no es la de efectuar declaraciones fácticas, sino más bien la de expresar nuestras emociones, en particular de aprobación o de rechazo" (Glock, 2012, p. 83), los juicios morales 
con relación a la justicia la reducen a lo justa o lo injusta que puede resultar una acción, y a una aprobación o rechazo fundamentado en criterios morales, ignorando la importancia que tiene la justicia desde la conciencia colectiva como virtud en la sociedad política.

Surgen dos condiciones intrínsecas de la justicia: la primera, que es una acción; y la segunda, que esa acción debe afectar a otro, puesto que ningún hombre se hace justicia a sí mismo. Entonces, la justicia es necesariamente una virtud política con una característica particular que es su indefinibilidad.

Si se sigue la tradición aristotélica de asimilar la justicia a la acción de hacer lo correcto, se cae en el error de asumir con un mismo grado de valor lo justo y la justicia, precisamente, el error de Aristóteles es no asumir la justicia desde la neutralidad y considerar, por el contrario, que su estudio debe hacerse desde virtudes éticas: "Aristóteles no piensa que la justicia sea neutral en esos términos. Cree que los debates sobre la justicia son, inevitablemente, debates acerca del honor, la virtud y la naturaleza humana" (Sandel, 2011, p. 212).

Lo curioso es que esos debates se dan en la actualidad, logrando no solo que la justicia se siga reduciendo a una acción individual, sino que el término se extienda a diferentes ámbitos de la cotidianeidad en relación con las virtudes. Por tanto, no existe la justicia en sí, sino la justicia en relación con un lugar, con una persona, con una comunidad o con un hecho, sumiéndose en un relativismo que imposibilita su apropiación. Para Maclntyre es claro que este posible relativismo presenta un problema no solo para la definición de justicia, sino para la definición de una sociedad justa: "este relativismo, cuando se combina con la opinión de que una virtud es la cualidad que conduce al éxito individual, envuelve a los que a ella se adhieren en un gran número de problemas consiguientes" (Maclntyre, 2001, p. 186).

Como se dijo, el problema de la filosofía moral en torno a la justicia es pretender reducirla a juicios éticos apoyados en las virtudes, pasando por alto que no existe una formula lógica que permita determinar que una acción bajo el influjo de las virtudes siempre producirá lo justo; tal sería el caso de una proposición como lo justo es $\mathrm{X}$ o hacer lo correcto es $\mathrm{Y}$, debido a que incluso en ocasiones lo justo es no hacer lo correcto.

Ahora, si no es posible definir la justicia por ser una cualidad simple ¿cómo entender la justicia? Esa es la tarea que le corresponde a la filosofía política de la mano con la filosofía analítica, erróneamente se ha creído que entre la una y la otra existe un tipo de incompatibilidad, sin embargo, la filosofía anglosajona ha demostrado, sobre todo desde el pensamiento de John Rawls y sus interlocutores, que para comprender el problema de la justicia es necesaria una filosofía que implique a la filosofía política y a la filosofía analítica. 


\section{Un resurgimiento en la filosofía política: Rawls y sus interlocutores}

En la obra A theory of justice (1971) John Rawls hace un resurgimiento de la justicia, entendiéndola como la primera virtud de las instituciones sociales. Para Rawls "puede pensarse que una concepción pública de la justicia constituye el rasgo fundamental de una asociación humana bien ordenada" (Rawls, 1995a, p. 19). De esta manera, la discusión de la justicia se traslada al campo de la filosofía política, ese es quizá el gran aporte de Rawls a la filosofía, así lo hace notar Van Parijs: "Y, de pronto, en 1971, con la aparición del libro de este profesor de Harvard que hasta entonces sólo había publicado algunos artículos dispersos, la filosofía política anglosajona experimentó un renacimiento espectacular" (1993, p. 49). Antes de Rawls, la reflexión de la justicia se hacía en el marco de las corrientes éticas, lo justo o lo injusto dependía de la concepción de la corriente, así, había diferencias en torno a la justicia, entre hedonistas, quienes consideran justo todo aquello que produzca placer, y utilitaristas, que consideran justo todo aquello que sea útil, ambas tenían al individuo como factor común.

Las dos doctrinas, mencionadas anteriormente, justifican la valoración de la justicia desde la esfera del individualismo, sin embargo, era necesario explicar la justicia desde la esfera de lo colectivo, que permitiera comprender la justicia no como un valor ético-moral, sino como un valor político. Rawls se encargará de esto al establecer dos principios de la justicia aplicables a cualquier sociedad política ordenada. De esta forma, Rawls saca la justicia del rango de la individualidad y la pone en el rango de la sociedad política, es decir, en el Estado. Para saber cuáles son los principios de justicia que regirán la sociedad política, Rawls retoma el concepto de estado de naturaleza de Locke donde hombres libres e iguales realizan el acuerdo inicial, y le agrega dos conceptos: la posición original y el velo de la ignorancia; a través de la aplicación de estos, asegura Rawls, se logrará un Estado imparcial, equitativo y garante de la justicia.

Con la posición original rawlsiana se pretende excluir información que podría influir en el proceso de deliberación acerca de los principios de justicia. El "velo de la ignorancia", que oculta a las partes rasgos característicos de sí mismos que enturbarían su razonamiento (Barry, 1997, pp. 30-31).

Sin embargo, ninguno de los dos conceptos - posición original y velo de la ignorancia- logra satisfacer completamente los presupuestos de una teoría de la justicia. Ante esta insatisfacción, y a fin de garantizar la duración del acuerdo inicial, Rawls propone un principio llamado "el principio de diferencia", según el cual es aceptable y tolerable recibir un trato desigual cuando se trata de mejorar las condiciones de los menos aventajados. Para Rawls, la justicia se sustenta en las condiciones y las posibilidades que los miembros de la sociedad política tienen para acceder a los bienes primarios y a los derechos fundamentales, esto se logra 
a través de la justicia distributiva, por eso, si al momento de distribuir los bienes y derechos en la sociedad, nadie sabe cuál será su posición final, el sujeto en la posición original tratará que esa distribución sea de la manera más equitativa e imparcial posible, a fin de no quedar en desventaja sea cual sea su posición final en la sociedad. Si esto no sucede, Rawls hace uso del principio de diferencia. De esta manera, la justicia distributiva es una rectificación inmediata de los errores que pudieron causarse en el acuerdo inicial, en la aplicación de la posición original y del velo de la ignorancia.

De lo anterior, se colige que la justicia como virtud pertenece a la sociedad política, entendiendo la sociedad política como garante de derechos, tales como la libertad y la igualdad, en ese sentido, aparecen la equidad y la imparcialidad como dos principios de la justicia en las sociedades políticas ordenadas, de manera que una vez establecida la distribución equitativa de los derechos y los bienes, estos deben ser garantizados en la sociedad política entendida como:

Un sistema equitativo de cooperación a lo largo del tiempo, de una generación a la siguiente, donde los que participan en la cooperación se conciben como ciudadanos libres e iguales y como miembros cooperativos normales de la sociedad durante toda su vida (Rawls, 2002, p. 26).

En toda sociedad política hay muchas cosas que se califican como justas o injustas, entre las que se encuentran las leyes, los sistemas políticos e incluso las acciones individuales, sin embargo, solo las leyes y los sistemas políticos necesitan legitimidad, la cual, según Ronald Dworkin, "depende de cómo ha adquirido un gobierno que se pretende tal su poder y de cómo lo usa" (2014, p. 392). La necesidad de legitimidad lleva al argumento de que solo de las instituciones básicas de la sociedad política es posible predicar la justicia, "por grandes instituciones entiendo la constitución política y las principales disposiciones económicas y sociales" (Rawls, 1995a, p. 20).

El problema que enfrenta Rawls es pretender dar respuesta desde la teoría a un problema de la práctica. Por ejemplo, que en una constitución se consagre expresamente la forma de distribución de derechos, obligaciones, deberes, garantías e incluso se preceptúen valores, tales como la solidaridad y la cooperación social, y que al leerla no quede duda de la creación de una sociedad justa, no es garantía suficiente para constituir una sociedad política justa, pues esta no responde per se a una constitución justa. Lo anterior se ajusta a lo dicho por Dworkin cuando al hablar de Rawls dice "John Rawls imagina a personas que negocian las condiciones de una constitución política original detrás de una cortina opaca que oculta a cada cual lo que él mismo realmente es, piensa y quiere" (2014, p. 428). Las palabras de Dworkin se asemejan a una de las críticas traídas por Brian Barry en La justicia como imparcialidad a la posición original, al tratarse de algo hipotético los acuerdos que de ahí resultan serán igualmente 
hipotéticos, enfrentándonos al problema de su aplicación en la realidad, es ahí donde se advierte que las personas más aventajadas no estarán en su mayoría dispuestas a soportar el principio de la diferencia. De manera que los hombres egoístas por naturaleza en el sentido Hobbesiano sacan a relucir ese egoísmo en la realidad.

Empero, Rawls insiste en que los hombres que han consentido el acuerdo inicial movidos por su racionalidad en la escogencia de los dos principios, los respetarán apelando al sentido de la justicia y convendrán unos mínimos de cooperación social. Rawls hace un esfuerzo en tratar de determinar los términos justos de la cooperación social, los cuales se basan en dos principios fundamentales ${ }^{3}$ : la libertad y la igualdad. Según Rawls, solo ciudadanos libres e iguales entre sí, pueden establecer un acuerdo de cooperación social, sin embargo, el mismo Rawls admite la existencia de la duda en dicho acuerdo tácito, "en la actualidad no existe ningún acuerdo sobre la forma en que las instituciones básicas de una democracia constitucional deben ordenarse si han de satisfacer los términos justos de cooperación entre ciudadanos considerados libres e iguales" (Rawls, 1995b, p. 30).

El principal argumento de Rawls consiste en afirmar que, en la medida que los individuos abandonen su carga moral y construyan un orden político justo, que garantice la participación de todos en la distribución de los bienes, es posible afirmar la existencia de una sociedad justa, sin embargo, dejamos en manos de individuos con cargas morales, sociales y políticas, la distribución de la riqueza y la preservación de la justicia.

Lo anterior resulta peligroso para el mismo objeto de la justicia, el cual resultaría reemplazado por acuerdos individuales, contrariando la esencia de la justicia como la primera virtud de las instituciones sociales, y se avocaría a una justicia parcializada. Para Robert Nozick, uno de los interlocutores más famosos de Rawls, cualquier fisura se puede presentar, por lo que la justicia distributiva podría conducir indirectamente a un relativismo moral de la justicia al quedar su administración en manos individuales, perdiendo su neutralidad,

Al escuchar el término "distribución", la mayoría de las personas supone que alguna cosa o mecanismo usa algún principio o criterio para hacer la distribución de cosas. Algún error pudo haberse colado en este proceso de distribución de las porciones (Nozick, 2017, p. 153).

\footnotetext{
${ }^{3}$ La exposición de estos dos principios es tratada por Rawls de manera diferente en Political Liberalism a como lo hizo en A theory of justice. Si bien pueden representarse bajo los valores de libertad e igualdad, la práctica de los principios exige unas condiciones especiales, el primer principio puede resumir que toda persona puede ejercer libremente sus derechos, en un esquema político que garantice el orden justo; el segundo principio solo soporta la desigualdad, si con ella se logra equilibrar la posición de los menos aventajados.
} 
Pero Nozick no es el único que ve este peligro en la justicia distributiva, Thomas Nagel también lo notó cuando en Igualdad y parcialidad criticó la justicia como imparcialidad propuesta por Rawls, en la medida que las instituciones sociales no han de ser totalmente imparciales, por el contrario, al ser administradas y operadas por los individuos, difícilmente estos se apartarán de sus concepciones morales y tomarán decisiones basadas en su carga moral. Aunque las instituciones sociales imponen una carga coercitiva, esta puede resultar no tan fuerte, frente a las concepciones morales individuales:

Las instituciones políticas sirven para algunos de los mismos propósitos que persiguen las convenciones morales, aunque nuestra participación en aquéllas, a diferencia de la obediencia a las exigencias morales, no es voluntaria sino que se nos impone coercitivamente (Nagel, 1996, pp. 2324).

Nagel tal vez haya pensado en los casos en que las instituciones sociales son utilizadas para el beneficio personal o de una minoría social, en contra de la sociedad política en general, lo que ocasiona que las instituciones sociales se alejen considerablemente de la cooperación social. A pesar de eso, Nagel no es claro al proponer una línea de acción que logre vencer la parcialidad a la que pueden verse avocados los ciudadanos como administradores de las instituciones sociales.

Para Michael Walzer, la crítica va más allá de la equidad e imparcialidad, pues admite la justicia distributiva cuando afirma que "el bien primario que distribuimos entre nosotros es el de la pertenencia en alguna comunidad humana" (1997, p. 44). Lo que será problemático es la forma de distribución, en ese sentido, para Walzer la justicia distributiva "guarda relación tanto como el ser y el hacer como con el tener, con la producción tanto como con el consumo, con la identidad y el status tanto como con el país, el capital o las posesiones personales" (p. 17).

De la propuesta Walzeriana se desata la inexistencia de una única vía de distribución de bienes, derechos, recursos, y demás, por lo tanto, no existe un solo modo de ser de la justicia distributiva. Efectivamente, ninguna sociedad política es absolutamente equitativa e imparcial, de modo que, al momento de distribuir derechos y bienes apela a unos argumentos fácticos para justiciar un trato diferenciable entre los individuos, esos argumentos de la justicia distributiva, como la cooperación y solidaridad, conducen al menos teóricamente al advenimiento de una sociedad política justa.

Para Rawls es claro que la justicia se concretiza, se materializa y se define en las instituciones sociales, y nunca en las acciones individuales. Alejar la justicia de la filosofía moral, donde corre el riesgo de sumirse en un relativismo, y traerla al 
campo de la filosofía política ha sido uno de los logros innegables de Rawls en la teoría de la justicia.

\section{La teoría de la acción como modo de adjetivación}

Para Hannah Arendt existen tres actividades fundamentales para la vita activa: labor, trabajo y acción. Sin embargo, de estas solo la acción puede ser considerada esencialmente política, ya que no puede pensarse fuera de la sociedad de los hombres,

La acción única actividad que se da entre los hombres sin la mediación de cosas o materia, corresponde a la condición humana de la pluralidad, al hecho de que los hombres, no el Hombre, vivan en la Tierra y habiten en el mundo (Arendt, 2009, pp. 21-22).

En el libro La condición humana, Arendt dedica el capítulo $\mathrm{V}$ a desarrollar la teoría de la acción. Para ella, lo natural del hombre es vivir en sociedad, esto es lo que permite la pluralidad desarrollada en dos actividades esenciales para el bios politikos aristotélico: la acción y el discurso, que constituyen elementos indispensables para la política.

La política se configura en la implicación de la acción y el discurso, para Arendt, el hombre no es naturalmente sino accidentalmente un ser político debido a que, si bien los hombres vienen dotados de la acción, algunos carecen de la capacidad de hacer vida en el discurso, es decir, les falta el segundo elemento propio de la política, de esta forma, no todo espacio público es inmediatamente un espacio político, ya que este surge siempre y en todo lugar cuando los hombres entran en contacto a través de la acción y la palabra.

Según Arendt, la política es una necesidad ineludible para la vida humana, tanto individual como social. Puesto que el hombre no es autárquico, sino que depende en su existencia de otros; el cuidado de la vida debe concernir a todos, sin esta la convivencia sería imposible. Arendt diferencia claramente el ser social del ser político en la sociedad u organización político-social, razón por la cual reprocha el zoon politikon aristotélico; para Hannah Arendt, todas las acciones humanas están condicionadas al hecho de vivir en comunidad, de modo que ninguna vida humana resulta posible sin la presencia de otros seres humanos, "ninguna clase de vida humana, ni siquiera la del ermitaño en la agreste naturaleza, resulta posible sin un mundo que directa o indirectamente testifica la presencia de otros seres humanos" (Arendt, 2009, p. 37). De esta manera, Arendt antepone en el hombre la vida social a la vida política, en cuanto que el hombre primero es naturalmente un animal socialis y no un zoon politikon. 
A pesar de considerarse al hombre como un animal socialis, la acción es un acto de decisión individual. "Por tanto, la pregunta «iqué debo hacer?» se refiere al comportamiento del yo con independencia de los otros" (Arendt, 2003, p. 44), no obstante, es imposible no coligarle que sus efectos recaigan sobre otros. Entendida la acción desde esta perspectiva de Arendt, es posible concebir la justicia como una acción que se realiza entre hombres, puesto que nadie es justo o injusto consigo mismo y se predica la justicia o la injusticia frente al otro "en cuanto a que el que obra injustamente es sólo injusto y no enteramente malo, no es posible ser injusto consigo mismo [...] porque por necesidad lo justo y lo injusto requieren más de una persona" (Aristóteles, Ética a Nicómaco, 2014).

La vida política es el reino de la acción, ahora, si concebimos la aplicación de la teoría de la justicia desde la perspectiva de la teoría de la acción como uno de los elementos esenciales para la vita activa, conducente a observar su incidencia en la sociedad política rawlsiana, nos encontraremos con que el primer modo de vida del hombre como ser social es la política y con ella la justicia como su fin excelso. Así, la justicia se constituye en la primera virtud de estructura básica de la sociedad, como lo afirma el mismo Rawls: "En una sociedad justa, las libertades de la igualdad de ciudadanía se dan por establecidas definitivamente; los derechos asegurados por la justicia no están sujetos a regateos políticos ni al cálculo de intereses sociales" (Rawls, 1995a, p. 17).

Toda sociedad política, por más homogénea que se pretenda, se sustentará en un grado alto de pluralidad. Y precisamente esta característica es el fundamento de la acción; de esta forma, la idea de Rawls de concebir la justicia como la primera virtud de la estructura básica de la sociedad política, llevaría a pensar en la existencia de una justicia para cada sociedad ya que, al estar la sociedad política fundada en la pluralidad y la pluralidad en los hombres, parecería lógico admitir que existen diferentes modos de ser de la justicia, de la misma forma que pasa con los hombres, sin que esto nos conduzca a un relativismo de la justicia; "los hombres se diferencian en vez de ser meramente distintos; son los modos en que los seres humanos se presentan unos a otros" (Arendt, 2009, p. 202).

La pluralidad de la sociedad humana es una condición indispensable para la realización del ser político del hombre desde la esfera pública, —que puede entenderse siguiendo a Jürgen Habermas - como un espacio donde los hombres interactúan a través de la comunicabilidad, realizando su vida social, creando la opinión pública y organizando el cuerpo público, "la pluralidad humana, básica condición tanto de la acción como del discurso, tiene el doble carácter de igualdad y distinción" (Arendt, 2009, p. 200). Precisamente, en Habermas el discurso adquiere la connotación de acción comunicativa, esto es, "la interacción de a lo menos dos sujetos capaces de lenguaje y de acción que (ya sea con medios verbales o con medios extraverbales) entablan una relación interpersonal" (Habermas, 1988, p. 124). 
La acción política desde el liberalismo constituye un ejercicio colectivo, ya que es la manifestación pública de la voluntad del pueblo, voluntad que se ve reflejada en la toma de decisiones que son vinculantes para todos, es decir, debe basarse en lo razonable sustentado en principios de validez y juicios racionales con pretensión de universalidad, esto fue considerado por Rawls como la razón pública, "característica de un pueblo democrático: es la razón de sus ciudadanos, de aquellos que comparten la calidad de ciudadanía en pie de igualdad" (Rawls, 1995b, p. 204). La igualdad ha de ser entendida como igualdad de derechos y libertades y no como igualdad de participación, es decir, como democracia formal y no activa.

Vemos que existe una similitud entre Arendt y Rawls, _aunque guardando cierto tipo de distancia- ambos presentan la igualdad como un principio fundante de la sociedad. Para Arendt, "si los hombres no fueran iguales, no podrían entenderse ni planear y prever para el futuro las necesidades de los que llegarán después" (Arendt, 2009, p. 200), estos elementos están claramente marcados en la posición original y en el velo de la ignorancia en la teoría de la justicia de Rawls. La concepción teórica de la acción implica para Arendt comenzar, es decir, tomar la iniciativa, por esta razón podemos adecuar la adjetivación de la justicia a la teoría de la acción, "el hecho de que el hombre sea capaz de acción significa que cabe esperarse de él lo inesperado" (Arendt, 2009, p. 202), esto sirve para comprender por qué un hombre recto puede cometer una acción injusta.

La adjetivación de la justicia desde la teoría de la acción, lleva a que cada hombre materialice el telos de la justicia. Debido a que "la acción sin un nombre, un «quién» unido a ella, carece de significado" (Arendt, 2009, pp. 205-206), el actuar del hombre estará en el centro de un resultado, el cual podrá ser justo o injusto y tendrá que responder por este juicio. Precisamente, al hablar de la acción, Arendt le otorga un elemento adicional, el poder, en ese sentido afirma:

el único factor material indispensable para la generación de poder es el vivir unido del pueblo. Sólo donde los hombres viven tan unidos que las potencialidades de la acción están siempre presentes, el poder puede permanecer con ellos, y la fundación de ciudades, que como ciudadesestado sigue siendo modelo para toda organización política occidental, es por lo tanto el más importante prerrequisito material del poder (Arendt, 2009, p. 224).

De modo que, frente a una injusticia estatal, solo el poder de los hombres unidos en comunidad puede lograr el cambio de las instituciones sociales, y generar una sociedad política justa. Las palabras de Arendt muestran que la finalidad de la acción, como uno de los fundamentos de la vita activa, está íntimamente relacionada con la facultad del hombre de vivir en sociedad, lo cual permite asumir la acción desde la justicia como justa o injusta, como el resultado propio entre hombres. 
Se parte del hecho de que los efectos son buenos o malos según el resultado que produzcan, sin embargo, lo justo o lo injusto podría tener una doble significación desde el sujeto activo y el sujeto pasivo de la acción, cabe entonces la pregunta: ¿puede una acción ser justa e injusta al mismo tiempo?, la respuesta afirmativa conduce a un relativismo que llevaría a la inexistencia de la justicia, por lo que es necesario examinarla desde lo que se ha denominado la adjetivación de la justicia.

Es importante aclarar que la pretensión de diferenciar las acciones humanas, según a quién o a quiénes afecte y no según a quién las realice, permite establecer a qué ámbito de la filosofía pertenece la justicia: si se asume lo justo o lo injusto como el resultado de una acción individual que produce efectos individuales, se estaría en el ámbito de la filosofía moral; pero si se asume como resultado de una acción colectiva que produce efectos colectivos, ya no se puede hablar de justo o injusto, sino de justicia o injusticia y eso hace parte del ámbito de la filosofía política.

Se puede entender como justo pagar las deudas, castigar a un hijo por una desobediencia, entre otras acciones, de esta manera, la justicia se presenta como un valor ético; sin embargo, lo ético no es la justicia sino su adjetivación, de modo que pagar las deudas no hace justicia, simplemente la acción se adjetivó en lo justo, del mismo modo que no se materializa la injusticia si no las pagara, sencillamente estaríamos frente a un acto de lo injusto.

Arendt da elementos que permiten concluir cómo su teoría de la acción, al partir del concepto de pluralidad y llegar al concepto de sociedad, da origen a la política, y con ella a todas las relaciones entre los hombres, donde la justicia como virtud es propia de la acción política, pero se adjetiva en la acción de cada individuo. "De ahí que todo agente, en tanto que hace, se deleita en hacer; puesto que todo lo que es apetece a su ser, y puesto que en la acción el ser del agente esta de algún modo ampliado" (Arendt, 1995, p. 104).

\section{A modo de conclusión}

A lo largo de la historia de la filosofía, el problema de la justicia ha tratado de adecuarse a un campo de estudio propio y determinado. Aunque en un principio se pensó que la filosofía moral era el ámbito, es claro que desde la publicación de la obra de John Rawls A theory of justice, el campo de discusión es la filosofía política. Esto basado en el planteamiento de George Moore de asumir lo bueno como concepto indefinible, y mi pretensión ha sido darle las mismas cualidades a la justicia, ya que solo nos acerca a ella un juicio de valor de justo o injusto, por lo que es lógico pensar que si se quiere una definición de justicia, ha de buscarse en la filosofía política. Para Rawls y sus interlocutores parece claro que la justicia 
pertenece al Estado como sociedad política; la discrepancia surge al determinar los principios de la justicia y en la forma como el Estado garantiza y aplica esos principios.

Teorizar la justicia ha sido una cuestión de la filosofía desde la época antigua, a pesar de eso, resulta siempre actual tratar de comprender en qué consiste eso de lo justo o determinar qué es eso que llamamos justicia, la discusión estará siempre abierta debido a lo cambiante de la forma de concebir tanto la ética como la política, la cual directamente repercute en la forma como asumimos lo justo y la justicia. Estamos ante sociedades cambiantes que exigen nuevos postulados éticos y políticos, y que hacen necesarios nuevos planteamientos sobre aquello que llamamos justo y sobre la justicia como valor político, que posibiliten responder a la pregunta iqué es una sociedad justa?, a partir de la comprensión de lo que es una teoría de la justicia; aclarando que no se trata del universalismo filosófico, sino del dinamismo que lleva a una constante reflexión de lo justo y la justicia como valor político.

Sin embargo, planteada la cuestión de la teoría de la acción y su papel en la interpretación de la adjetivación de la justicia, queda por determinar a cuál sociedad política corresponde la justicia como valor y cuáles serán los principios o postulados que marcarán la ruta a seguir, entendiendo que la adjetivación de la justicia es predicable incluso en sociedades políticas no democráticas. Permanece la duda: ipuede asumirse la justicia desde la reflexión filosófica como un valor exclusivo de las sociedades políticas democráticas?

\section{Referencias}

Arendt, H. (1995). De la historia a la acción. Barcelona: Paidós.

Arendt, H. (1997). ¿Qué es la política? Barcelona: Paidós.

Arendt, H. (2003). Conferencias sobre la filosofía política de Kant. Buenos Aires: Paidós.

Arendt, H. (2009). La condición humana. Barcelona: Paidós.

Aristóteles (2014). Ética a Nicómaco. Madrid: Gredos.

Barry, B. (1997). La justicia como imparcialidad. Barcelona: Paidós.

Dworkin, R. (2014). Justicia para erizos. Buenos Aires: Fondo de Cultura Económica. 
Foot, P. (2002). Bondad Natural. Barcelona: Paidós.

Glock, H. J. (2012). ¿Qué es la filosofía Analítica? Madrid: Tecnos.

Habermas, J. (1988). Teoría de la acción comunicativa, I. Madrid: Taurus.

Kelsen, H. (2014). ¿Qué es la justicia? (A. Calsamiglia, trad.). Medellín: Editorial Universidad de Antioquia.

MacIntyre, A. (1994). Justicia y Racionalidad. Barcelona: Ediciones Internacionales Universitarias.

MacIntyre, A. (2001). Tras la virtud. Barcelona: Crítica.

Moore, G. E. (1903). Principia Ethica. Cambridge: Cambridge University Press.

Nagel, T. (1996). Igualdad y parcialidad. Barcelona: Paidós.

Nozick, R. (2017). Anarquía, Estado y utopía. Ciudad de México: Fondo de Cultura Económica.

Rawls, J. (1971). A theory of justice. Cambridge: Harvard University Press.

Rawls, J. (1995a). Teoría de la justicia. Ciudad de México: Fondo de Cultura Económica.

Rawls, J. (1995b). Liberalismo político. Ciudad de México: Fondo de Cultura Económica.

Rawls, J. (2002). La justicia como equidad. Barcelona: Paidós.

Sandel, M. J. (2011). Justicia. ¿Hacemos lo que debemos? Barcelona: Debate.

Van Parijs, P. (1993). ¿Qué es una sociedad justa? Barcelona: Ariel.

Walzer, M. (1997). Las esferas de la justicia. Ciudad de México: Fondo de Cultura Económica. 